\title{
Alternatively activated macrophages are associated with metastasis and poor prognosis in prostate adenocarcinoma
}

\author{
WENXUE HU, YUNJUAN QIAN, FENG YU, WEI LIU, YANHUA WU, XIAOWU FANG and WENKE HAO \\ Guangdong Academy of Medical Science, Guangdong General Hospital, Guangdong Provincial Institute of Geriatrics, \\ Guangzhou, Guangdong 510080, P.R. China
}

Received August 19, 2014; Accepted May 7, 2015

DOI: $10.3892 / \mathrm{ol} .2015 .3400$

\begin{abstract}
Recent studies have revealed that alternatively activated macrophages (AAMs) are involved in tumor progression. However, the effect of AAMs on the metastasis of prostate cancer is poorly understood. In the present study, the prostate tissues of 42 patients with prostate adenocarcinoma $(\mathrm{PCa})$ were used in the analysis of tumor associated macrophages (TAMs) and AAMs by immunofluorescence. The patients were followed up for 5 years. The associations of TAMs and AAMs with the clinicopathological features and outcome in these cases were evaluated. Immunofluorescent analysis indicated that the mean number of TAMs (CD68-positive cells) in the prostate tissues of PCa patients with metastasis $[45.29 \pm 7.25$ cells/high-power field (HPF)] was significantly higher compared with that of $\mathrm{PCa}$ patients without metastasis $(33.57 \pm 5.25$ cells $/ \mathrm{HPF}$; $\mathrm{P}<0.01)$. The mean numbers of AAMs (CD68- and CD206-positive cells) in the tissues of PCa patients with and without metastasis were $29.43 \pm 5.68$ and $9.14 \pm 5.29$ cells/HPF, respectively. In addition, the percentage of AAMs (number of AAMs/number of TAMs) was $65.11 \pm 9.68$ and $27.32 \pm 7.85 \%$ in patients with and without metastasis, respectively. The differences in the number and percentage of AAMs between the two groups were statistically significant $(\mathrm{P}<0.01)$. The number and percentage of AAMs was positively correlated with tumor grade and serum prostate-specific antigen (PSA) level. Univariate analysis indicated that the level of PSA, Gleason score, metastatic status, $\mathrm{T}$ grade, number of TAMs, number of AAMs and percentage of AAMs were predictors of the overall survival. Furthermore, multivariate analyses revealed that Gleason score, level of PSA and number of TAMs were predictors for overall survival rate. These results indicate that TAMs and
\end{abstract}

Correspondence to: Professor Wenke Hao, Guangdong Academy of Medical Science, Guangdong General Hospital, Guangdong Provincial Institute of Geriatrics, Building no. 106, 2 Zhongshan Road, Guangzhou, Guangdong 510080, P.R. China

E-mail: hwk1964@sina.com

Key words: alternatively activated macrophage, metastasis, prognosis, prostate adenocarcinoma, tumor associated macrophage
AAMs may be important in the metastasis of $\mathrm{PCa}$, and that TAMs and AAMs may be used as potential biomarkers of poor prognosis in late-stage PCa patients.

\section{Introduction}

Prostate cancer is one of the most common malignancies in male patients of advanced age and is the second leading cause of cancer-associated mortality worldwide $(1,2)$. The incidence of prostate cancer has increased in China in recent years (3), and the majority of cases is diagnosed in the metastatic stages of the disease, and have thus lost the opportunity for radical surgery $(4,5)$. The use of prostate cancer biomarkers, including the prostate-specific antigen (PSA), and Gleason scores has revolutionized the screening, detection and determination of prognosis of this disease (6). It would be informative to specifically block immune biomarkers or molecules which could promote the progression of PCa. However, reliable biomarkers associated with the immune response of $\mathrm{PCa}$ are not established at present (7). Therefore, identification of immune biomarkers of invasion and metastasis is required for the diagnosis and prognosis of patients with prostate cancer.

Macrophages are the most abundant cancer stromal cells involved in the host immune system (8). Previous studies have reported that tumor-associated macrophages (TAMs) originate from circulating monocytes and are a key component of the inflammatory microenvironment (9-11). TAMs are recruited and maintained in neoplastic tissues by various chemokines and cytokines. Recent evidence also suggests that TAMs may be involved in cancer progression as they release cytokines, extracellular matrix proteins and growth factors [including transforming growth factor (TGF)- $\beta$ and vascular endothelial growth factor] that promote proliferation, angiogenesis, and metastasis (9-11). In a number of cancer types, including breast, colon and bladder cancer, TAM infiltration has been found to be associated with poor outcome (12-14).

Macrophages differentiate into functionally distinct immunological populations depending on the cy tokine environment. Classically activated macrophages (CAMs) are considered to be induced by interferon $\gamma$ and lipopolysaccharides, which function predominantly in inflammation, tissue damage and the killing of intracellular microbes (15-17). However, alternatively activated macrophages (AAMs) are induced under the influence of T-helper type 2 cytokines, particularly 
interleukin (IL)-4 and IL-13. This phenotype features the induction of arginase 1 and upregulates the expression of surface molecules, including CD206 (also known as mannose receptor), and scavenger receptors (CD163) (14). Notably, this particular macrophage subset is the predominant source of cytokines and chemokines, including IL-10, TGF- $\beta$ and chemokine C-C motif ligand 18 (CCL18). Through such cytokine pathways, AAM may be involved in the downregulation of inflammation, tissue remodeling and elimination of tissue debris and apoptotic bodies, as well as tumor progression (15-18).

Certain studies have demonstrated that the number and density of AAMs in cancer tissues is highly increased when compared with normal tissues, and is associated with the prognosis. In addition, two recent studies have indicated that TAMs and AAMs participated in the progression of prostate cancer (19-21). However, the association between differentiation of macrophages and metastasis of prostate adenocarcinoma (PCa) is not well-established. Therefore, we hypothesized that AAMs may contribute to the metastasis of PCa and are associated with prognosis. In the present study, TAMs and AAMs were analyzed in prostate tissues of PCa by immunofluorescence. In addition, the association of TAMs and AAMs with the clinicopathological features and outcome in these patients were investigated.

\section{Patients and methods}

Patients. Between January 2008 and March 2009, a total of 42 patients with PCa underwent surgery or needle aspiration biopsy with diagnostic or curative intent in Guangdong General Hospital (Guangzhou, China). The patients were followed up for 5 years in order to obtain a complete set of clinical data. The inclusion criteria for the study was: i) Patients who were diagnosed with PCa with or without metastasis; ii) aged between 60 and 85 years old; and iii) patients who fit the above criteria who agreed to sign informed consents. Each patient was treated according to their stage of disease. The patients had not received any form of therapy before surgery. Radical resection (Ransurethral plasmakinetic resection and testes resection) was used in T1-T2 PCa patients. All the patients received androgen deprivation therapy using a hormone-releasing hormone agonist analogue. Tumors were confirmed histopathologically and were staged according to the AJCC/UICC TNM System (22). The histological types were determined by two independent clinical pathologists in a double-blinded manner: all the samples were $\mathrm{PCa}$. The samples were collected were according to the status of metastasis, following treatment. A total of 8 patients diagnosed with benign prostatic hyperplasia were used as the control group. The study involving human participants was approved by the Ethics Committee of Guangdong General Hospital. Written informed consent was obtained from the patients prior to enrollment.

Histology and immunofluorescence. Prostate tissues of PCa patients were collected by surgery or needle aspiration biopsy and fixed in paraformaldehyde for $12 \mathrm{~h}$. Paraffin-embedded sections $(8 \mu \mathrm{m})$ were cut and stained with hematoxylin and eosin (Boster Biological Technology Ltd., Wuhan, China) for pathological diagnosis. For confocal imaging analysis, paraffin-embedded sections $(8 \mu \mathrm{m})$ were cut and dehydrated in xylene and a graded alcohol series, followed by antigen retrieval; Briefly, the sections were immersed in citrate buffer with pH 6.0 in a specialized pressure cooker designed for antigen retrieval (Head Biotechnology Ltd., Beijing, China). When the cooker reached a temperature of $120^{\circ} \mathrm{C}$ and pressure of $117 \mathrm{KPa}$, the sections were boiled for $5 \mathrm{~min}$, then cooled down in the cooker for 3-4 h. The sections were blocked with $1 \%$ bovine serum albumin (Boster Biological Technology, Ltd., Wuhan, China) and normal goat serum (Boster Biological Technology Company) for $30 \mathrm{~min}$ at $37^{\circ} \mathrm{C}$, and then incubated with a mouse monoclonal antibody against CD68 (ab955; Abcam, Cambridge, UK; dilution, 1:100) and a rabbit polyclonal antibody against CD206 (ab64693; Abcam; dilution, 1:50) overnight at $4^{\circ} \mathrm{C}$. Subsequently, the sections were washed in phosphatebuffered saline (PBS), and incubated with Alexa Fluor ${ }^{\circledR} 488$ goat anti-rabbit IgG (A-24922; Invitrogen Life Technologies, Carlsbad, CA, USA; dilution, 1:1,000) and Alexa Fluor ${ }^{\circledR} 633$ goat anti-mouse IgG (A-21052; Invitrogen Life Technologies; dilution, $1: 1000$ ) at $37^{\circ} \mathrm{C}$ for $30 \mathrm{~min}$. Next, the sections were washed in PBS, incubated with 4',6-diamindino-2-phenylindole dihydrochloride (Sigma-Aldrich, St. Louis, MO, USA; dilution, 1:100) for nuclear staining, sealed with aqueous mounting medium (R\&D Systems, Inc., Minneapolis, MN, USA), and viewed under a Zeiss LSM 510 confocal imaging system (Carl Zeiss AG, Dresden, Germany). Five fields of cancer cores from each section were randomly selected and viewed; then, the number of CD68-positive cells and CD68/CD206-doublepositive cells were counted.

Statistical analysis. All the obtained numerical data are expressed as the mean \pm standard deviation. Comparisons between groups were performed using an independent samples t-test and $\chi^{2}$ test. The association of TAMs and AAMs with clinical characteristics was assessed using Pearson's correlation coefficient. Overall survival was measured from the initiation of diagnosis until the end of the observation period and analyzed using the Kaplan-Meier method. Cox proportional hazards analysis using a univariate or multivariate method was used to explore the effect of variables on overall survival. All the statistical calculations were processed using SPSS version 16.0 software (SPSS, Inc., Chicago, IL, USA). P $<0.05$ was considered to indicate a statistically significant difference.

\section{Results}

Clinical features of PCa patients. A total of 42 patients (21 with metastasis and 21 without metastasis) diagnosed with prostate adenocarcinoma were recruited. PCa with metastasis was defined as invasion of other organs, including bone, visceral (such as bladder, testes, and other organs) or lymph nodes. To assess if the PCa patients were metastatic, bone scans, ultrasound, MRI scans (or CT or PET scans) were conducted. Age and survival time did not differ significantly between patients with and without metastasis. Patients with metastasis possessed significantly higher PSA levels, Gleason scores and tumor grades (Table I).

Identification of TAMs and AAMs in prostate tissues of $P C a$ patients with or without metastasis. Immunofluorescence analysis revealed that the mean number of TAMs (CD68-positive 
Table I. Clinical features of prostate adenocarcinoma patients with or without metastasis.

\begin{tabular}{|c|c|c|c|}
\hline & Metastasis & Non-metastasis & P-value \\
\hline Age, years & $70.05 \pm 10.05$ & $73.00 \pm 7.76$ & $P>0.05$ \\
\hline PSA, ng/ml & $87.29 \pm 48.12$ & $16.33 \pm 7.15$ & $\mathrm{P}<0.01$ \\
\hline Gleason scores & $7.24 \pm 1.04$ & $6.587 \pm 0.96$ & $\mathrm{P}<0.05$ \\
\hline Tumor grade $(\mathrm{T}), \mathrm{n}$ & & & $\mathrm{P}<0.01$ \\
\hline $1-2$ & 0 & 18 & \\
\hline $3-4$ & 21 & 3 & \\
\hline Survival time, $\mathrm{n}$ & & & $\mathrm{P}>0.05$ \\
\hline$<5$ years & 8 & 4 & \\
\hline$\geq 5$ years & 13 & 17 & \\
\hline
\end{tabular}

Data regarding age, PSA and Gleason scores are presented as the mean \pm standard deviation. PSA, prostate-specific antigen.

Table II. Correlation between macrophages and clinical features.

\begin{tabular}{lcr}
\hline Parameters & Pearson correlation coefficient & P-value \\
\hline Number of TAMs vs. Gleason scores & 0.723 & P $<0.01$ \\
Number of AAMs vs. Gleason scores & 0.848 & P $<0.01$ \\
Percentage AAMs vs. Gleason scores & -0.520 & $P<0.05$ \\
Number of TAMs vs. level of PSA & 0.418 & $\mathrm{P}<0.05$ \\
Number of AAMs vs. level of PSA & 0.746 & $\mathrm{P}>0.05$ \\
Percentage of AAMs vs. level of PSA & -0.713 & $\mathrm{P}<0.01$ \\
\hline
\end{tabular}

TAM, tumor-associated macrophage; AAM, alternatively activated macrophage; PSA, prostate-specific antigen.

Table III. Univariate and multivariate Cox proportional HRs for overall survival.

\begin{tabular}{|c|c|c|}
\hline Characteristic & $\operatorname{HR}(95 \% \mathrm{CI})$ & P-value \\
\hline \multicolumn{3}{|l|}{ Univariate analysis } \\
\hline Age, years & $1.017(0.952-1.086)$ & $\mathrm{P}>0.05$ \\
\hline PSA level & $1.022(1.011-1.033)$ & $\mathrm{P}<0.01$ \\
\hline Gleason score & $4.014(2.040-7.899)$ & $\mathrm{P}<0.01$ \\
\hline Tumor grade & $2.538(1.178-5.467)$ & $\mathrm{P}<0.05$ \\
\hline Treatment $^{\mathrm{a}}$ & $0.722(0.229-2.278)$ & $\mathrm{P}>0.05$ \\
\hline Metastatic status & $12.888(1.659-100.096)$ & $\mathrm{P}<0.05$ \\
\hline Number of TAMs & $1.089(1.030-1.151)$ & $\mathrm{P}<0.01$ \\
\hline Number of AAMs & $1.182(1.091-1.281)$ & $\mathrm{P}<0.01$ \\
\hline Percentage of AAMs (AAMs/TAMs) & $0.053(0.070-0.411)$ & $\mathrm{P}<0.01$ \\
\hline \multicolumn{3}{|l|}{ Multivariate analysis } \\
\hline PSA level & $1.025(1.002-1.051)$ & $\mathrm{P}<0.05$ \\
\hline Gleason scores & $2.579(0.481-13.836)$ & $\mathrm{P}<0.05$ \\
\hline Tumor grade & $0.983(0.732-1.011)$ & $\mathrm{P}>0.05$ \\
\hline Metastatic status & $1.230(1.030-1.533)$ & $\mathrm{P}>0.05$ \\
\hline Number of TAMs & $1.524(1.155-2.012)$ & $\mathrm{P}<0.01$ \\
\hline Number of AAMs & $0.838(0.677-1.039)$ & $P>0.05$ \\
\hline Percentage of AAMs (AAMs/TAMs) & $1.531(0.134-17.545)$ & $\mathrm{P}>0.05$ \\
\hline
\end{tabular}

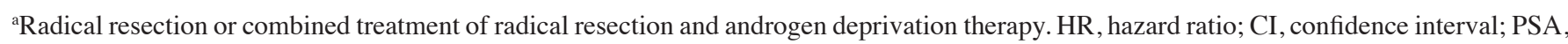
prostate-specific antigen; TAM, tumor-associated macrophage; AAM, alternatively activated macrophage. 
A
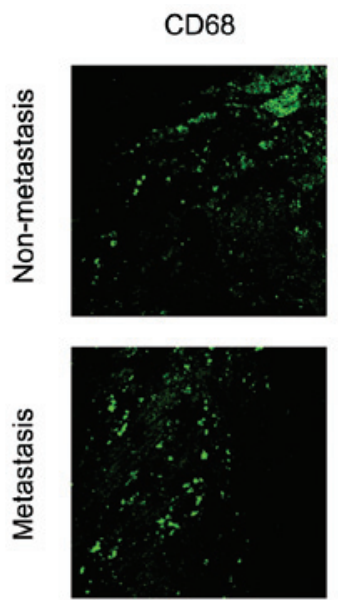

CD206
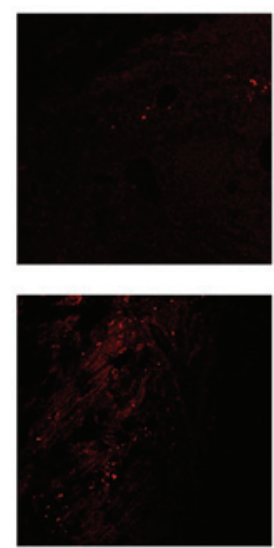

DAPI
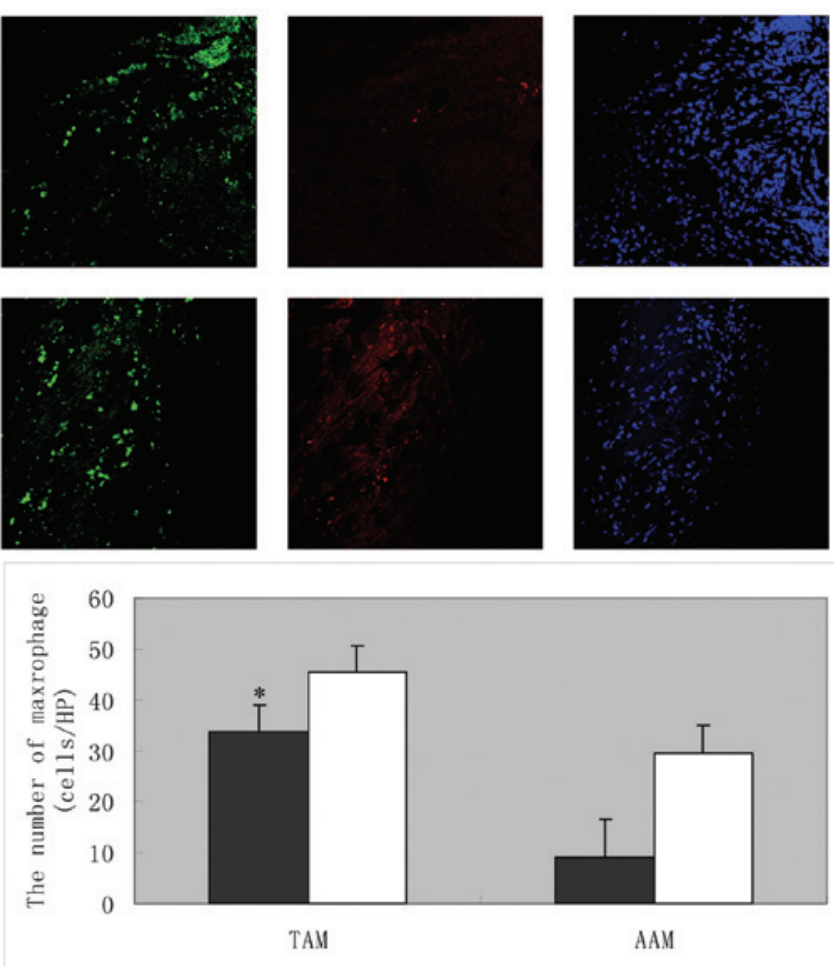

Merge
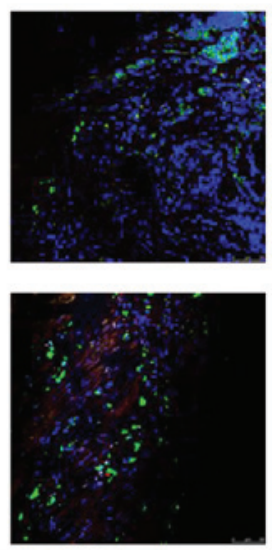

Non-metastasis $\square$ Metastasis

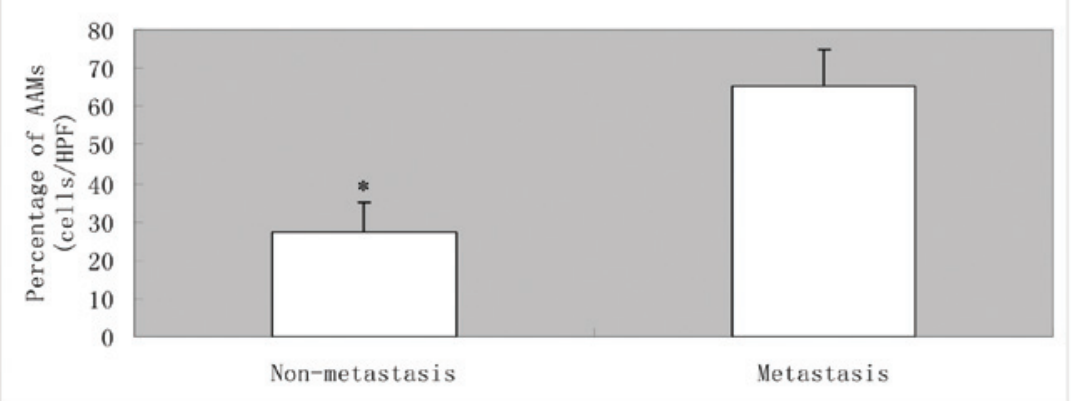

Figure 1. AAMs in prostate tissues of PCa patients with or without metastasis. (A) Confocal photomicrographs (magnification, x800) reveal the number of TAMs (CD68-positive cells) and AAMs (co-localization signals of CD68 and CD206) are significantly higher than that of PCa patients without metastasis. Blue corresponds to nuclear staining, green corresponds to CD68 staining and red corresponds to CD206 staining. (B) Number and percentage of AAMs in prostate tissues of PCa patients. Results are presented as the mean \pm standard error of the mean for a group of 21 patients. ${ }^{*} \mathrm{P}<0.01$. PCa, prostate adenocarcinoma; TAM, tumor-associated macrophage; AAM, alternatively activated macrophage; HPF, high-power field.

cells) in the prostate tissues of PCa patients with metastasis [45.29 7.25 cells/high power field (HPF)] was significantly higher compared with that of PCa patients without metastasis (33.57 \pm 5.25 cells/HPF; $\mathrm{P}<0.01)$. Co-localization signals of CD68 and CD206 were identified as AAMs, and the numbers of AAMs in PCa patients with and without metastasis were $29.43 \pm 5.68$ cells/HPF and $9.14 \pm 5.29$ cells/HPF, respectively (Fig. 1). The mean percentage of AAMs (calculated as the number of AAMs / number of TAMs) in patients with and without metastasis were $65.11 \pm 9.68$ and $27.32 \pm 7.85 \%$, respectively. The differences in the number and percentage of AAMs between these patient groups were statistically significant $(\mathrm{P}<0.01)$.

Association of TAMs and AAMs with the Gleason score and level of PSA. Pearson's correlation coefficient analysis revealed that the numbers of AAMs and TAMs were significantly positively correlated with the Gleason scores of the patients $(\mathrm{P}<0.01$; Table II). The number of TAMs and the level of serum PSA were also found to be significantly correlated $(\mathrm{P}<0.05)$. The percentage of AAMs was found to be negatively correlated with Gleason scores and PSA levels. However, the numbers of AAMs were not correlated with the level of serum PSA.

Association of TAMs and AAMs with the percentage of AAMs and survival. To assess the association between levels of TAMs, AAMs and patients' overall survival time, patients were followed up for $>5$ years. Higher levels of TAMs and AAMs, and higher percentages of AAMs were associated with shorter overall survival time, as demonstrated by the Kaplan-Meier analysis (Fig. 2). Univariate and multivariate analyses were also performed separately to analyze several variables as predictors of $\mathrm{PCa}$. Univariate analysis results indicated that 
A

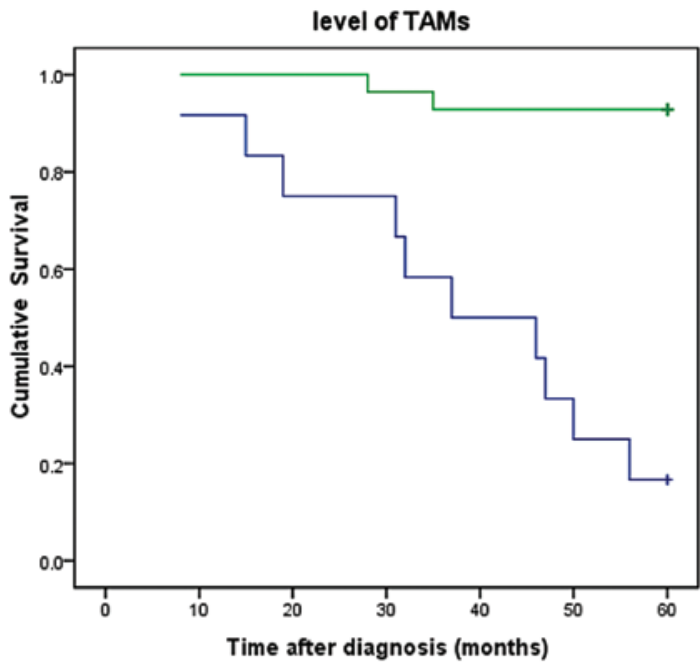

B

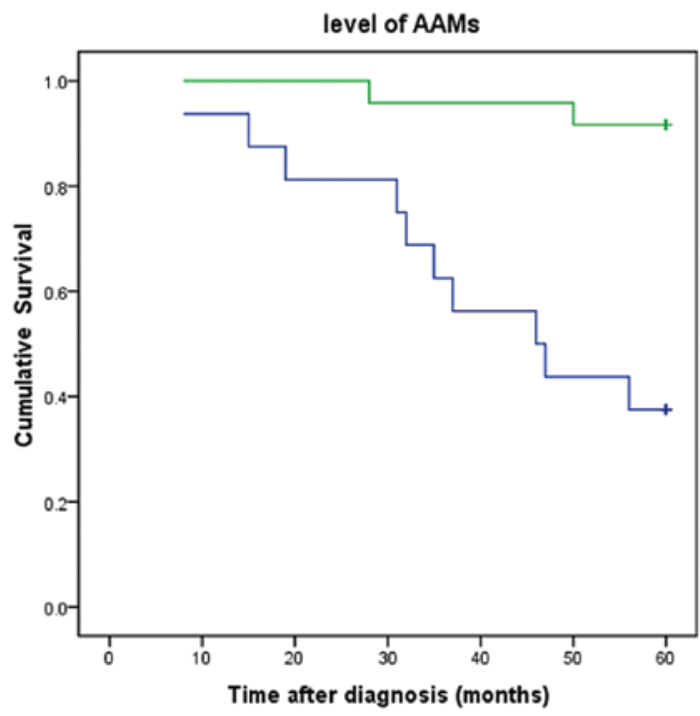

C

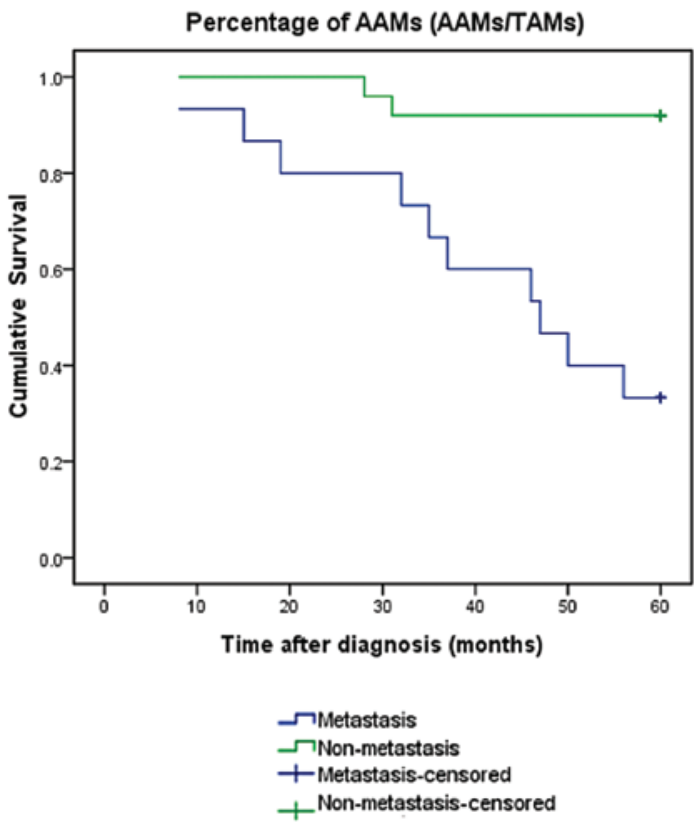

Figure 2. Kaplan-Meier analysis of overall survival. Survival curves demonstrate the association between overall survival following diagnosis and the (A) level of TAMs, (B) level of AAMs and (C) percentage of AAMs. Statistically significant differences between the groups were estimated using $\chi^{2}$ test. TAM tumor-associated macrophage; AAM, alternatively activated macrophage. the PSA levels, Gleason scores, metastatic status, T grade, number of TAMs, number of AAMs and percentage of AAMs are predictive factors for the overall survival of patients. Multivariate analyses indicated that the Gleason scores, PSA levels and number of TAMs are predictive factors for overall survival (Table III). Thus, high TAM and AAM levels may be used as new markers to predict poor prognosis among patients with late-stage PCa.

\section{Discussion}

The current study demonstrated that the number of TAMs and AAMs were significantly increased in PCa tissues of patients with metastasis compared with that of patients without metastasis. Furthermore, the number of AAMs and TAMs were positively correlated Gleason scores, and the number of TAMs were correlated with PSA levels. Additionally, higher levels of TAMs, levels of AAMs and percentages of AAMs were associated with shorter overall survival. In addition, univariate analysis indicated that the level of PSA, Gleason scores, metastasis status, T grade, number of TAMs, number of AAMs and percentage of AAMs are predictors for overall survival, whilst multivariate analyses demonstrated that Gleason scores, PSA level and number of TAMs are predictors for overall survival.

Recent studies have demonstrated that TAMs play a critical biological role in prostate cancer initiation and progression (10-21). Gollapudi et al (20) demonstrated that TAM levels were higher in prostatic intraepithelial neoplasia compared with the levels in benign tissue, while patients with higher Gleason scores contained higher TAM numbers than those with lower Gleason scores. Similarly, Herroon et al (23) presented evidence that bone marrow macrophages, supplying the cysteine protease cathepsin $\mathrm{K}$, may be involved in CCL2- and cyclooxygenase-2-driven pathways that contributed to tumor progression in the bone. The present study also demonstrated that the number of TAM (CD68-positive) cells in prostate tissues of $\mathrm{PCa}$ patients with metastasis was significantly higher compared with the number in PCa patients without metastasis. This indicates that TAMs play an important role in the progression of metastasis in PCa patients.

Macrophages are activated towards the alternatively activated phenotype by stimulation with IL-4 and IL-13 $(24,25)$. Several studies have suggested that AAMs promote tumor growth, angiogenesis and invasion (26-28). However, the role of AAMs in metastasis of PCa was previously undefined. Lanciotti et al (19) collected clinical and pathological data from 93 patients treated with radical prostatectomy and analyzed the association between CAMs and AAMs occurrence. They concluded that a higher density of macrophages was associated with poor prognosis and that AAM was significantly associated with tumor extension. Furthermore, Comito et al (21) demonstrated that $\mathrm{PCa}$ cells participate in the differentiation process through secretion of monocyte chemotactic protein-1, facilitating monocyte recruitment, macrophage differentiation and M2 polarization. This complex interplay among cancer cells and AAMs contributes to increasing tumor cell motility, ultimately facilitating the escape of cancer cells from the primary tumor and metastatic spread; therefore, Comito et al (21) concluded that AAMs interact with cancerassociated fibroblasts during prostate carcinoma progression. 
The present study investigated the function of AAMs on PCa in a cellular or molecular level. The current results demonstrated that the number of AAMs in PCa patients with metastasis was significantly higher compared with that in patients without metastasis. In addition, the percentage of AAMs (number of AAMs/number of TAMs) was significantly higher in $\mathrm{PCa}$ patients with metastasis. The collective findings of the aforementioned studies and the present study suggest that AAMs may promote the metastasis of PCa.

The present findings also revealed that numbers of AAMs and TAMs are significantly positively correlated with Gleason scores. The analysis of the TAM number and serum PSA level also revealed a significant correlation. Numerous studies have demonstrated that Gleason scores and PSA levels are associated with the severity of clinical outcome. Huang et al (29) reported that the initial PSA level, a PSA nadir of $\geq 2 \mathrm{ng} / \mathrm{ml}$ and shorter time to PSA nadir were associated with disease progression. Furthermore, Chen et al (30) showed that initial level of PSA was a potential predictive factor for biochemical progression. Petrylak et al (31) demonstrated that greater PSA declines were associated with survival in the Southwest Oncology Group (SWOG) trial. Another study demonstrated that a PSA decline of $\geq 30 \%$ within 3 months of chemotherapy initiation had the highest degree of surrogacy for overall survival, confirming the results of the SWOG trial (32). These clinical trials indicated that the initial PSA level and PSA declines were associated with prognosis. The biopsy Gleason scores, defined as the sum of the most predominant Gleason grades, is a prognostic factor significantly associated with the risk of prostate cancer-specific mortality (PCSM) following conservative or curative management $(33,34)$. Studies have established an association between the commonly used Gleason score levels (8-10 vs. 7 vs. $\leq 6)$ and an increased risk of PCSM after adjusting for known prognostic factors $(35,36)$. These data may suggest that AAM and TAM levels were consistent with the clinical features such as the levels of PSA and the Gleason scores. However, the number of AAMs was not correlated with the level of serum PSA. This phenomenon may be due to the wide range of PSA variation.

Kaplan-Meier analysis revealed that higher TAM and AAM numbers, and higher percentages of AAMs were associated with shorter overall survival. This indicates that TAMs and AAMs may be closely connected with the prognosis of PCa. The combination of TAM and AMM numbers, and percentage of AAMs is, therefore, a sensitive marker for poor prognosis. In addition, the level of PSA, Gleason scores, metastatic status and clinical stage of disease were found to be significantly predictive of overall survival by univariate and multivariate analyses. The classical prognostic factors of clinical stage, such as level of PSA and Gleason scores, have been used for over a decade to categorize patients at the time of diagnosis into broad risk groups that help to determine appropriate management strategies (33-36). The present results are consistent with these factors. Finally, univariate analysis demonstrated that the number of TAMs, number of AAMs and percentage of AAMs are predictors for overall survival, whilst multivariate analyses indicated that the number of TAMs is a predictor for overall survival. Thus, these data indicated that high levels of TAMs and AAMs may be used as a pathological and immune marker to predict poor prognosis among patients with late-stage PCa in addition to Gleason scores. However, the number of AAMs was not found to be a predictor of the overall survival based on multivariate analysis. This may be due to the particular samples used in the current study, and more cases should be recruited in further studies.

In conclusion, the results demonstrated that AAMs may be important in the metastasis of PCa and may be used as potential immune biomarkers of poor prognosis in patients with late-stage PCa. However, the underlying mechanism of AAMs in the metastasis of $\mathrm{PCa}$ and how this may be blocked remain to be established and require further investigation.

\section{Acknowledgements}

This study was supported by a grant from the Medical Scientific Research Foundation of Guangdong Province, China (no. B007).

\section{References}

1. Collin SM, Martin RM, Metcalfe C, et al: Prostate-cancer mortality in the USA and UK in 1975-2004: An ecological study. Lancet Oncol 9: 445-452, 2008.

2. Siegel R, Naishadham D and Jemal A: Cancer statistics, 2013. CA Cancer J Clin 63: 11-30, 2013.

3. Han SJ, Zhang SW, Chen WQ and Li CL: Analysis of status and trends of prostate cancer incidence in China. Lin chuang Zhong Liu Xue Za Zhi 18: 330-335, 2013.

4. Tammela T: Endocrine treatment of prostate cancer. J Steroid Biochem Mol Biol 92: 287-295, 2004.

5. Loblaw DA, Virgo KS, Nam R, et al; American Society of Clinical Oncology: Initial hormonal management of androgen-sensitive metastatic, recurrent, or progressive prostate cancer: 2006 update of an American Society of Clinical Oncology practice guideline. J Clin Oncol 25: 1596-1605, 2007.

6. Phillips JG, Aizer AA, Chen MH, Zhang D, Hirsch MS, Richie JP, Tempany CM, Williams S, Hegde JV, Loffredo MJ and D'Amico AV: The effect of differing Gleason scores at biopsy on the odds of upgrading and the risk of death from prostate cancer. Clin Genitourin Cancer 12: e181-187, 2014.

7. Mohammed AA: Biomarkers in prostate cancer: New era and prospective. Med Oncol 31: 140, 2014.

8. Chiarugi P, Paoli P and Cirri P: Tumor microenvironment and metabolism in prostate cancer. Semin Oncol 41: 267-280, 2014.

9. Gollapudi K, Galet C, Grogan T, et al: Association between tumor-associated macrophage infiltration, high grade prostate cancer and biochemical recurrence after radical prostatectomy. Am J Cancer Res 3: 523-529, 2013.

10. Schmieder A, Michel J, Schönhaar K, Goerdt S and Schledzewski K: Differentiation and gene expression profile of tumor-associated macrophages. Semin Cancer Biol 22: 289-297, 2012.

11. Solinas G, Schiarea S, Liguori M, et al: Tumor-conditioned macrophages secrete migration-stimulating factor: A new marker for M2-polarization, influencing tumor cell motility. J Immunol 185: 642-652, 2010.

12. Su S, Liu Q, Chen J, et al: A positive feedback loop between mesenchymal-like cancer cells and macrophages is essential to breast cancer metastasis. Cancer Cell 25: 605-620, 2014.

13. Takayama H, Nishimura K, Tsujimura A, et al: Increased infiltration of tumor associated macrophages is associated with poor prognosis of bladder carcinoma in situ after intravesical bacillus Calmette-Guerin instillation. J Urol 181: 1894-1900, 2009.

14. Zhou Q, Peng RQ, Wu XJ, et al: The density of macrophages in the invasive front is inversely correlated to liver metastasis in colon cancer. J Transl Med 8: 13, 2010.

15. Gordon S: Alternative activation of macrophages. Nat Rev Immunol 3: 23-35, 2003.

16. Martinez FO, Sica A, Mantovani A and Locati M: Macrophage activation and polarization. Front Biosci 13: 453-461, 2008.

17. Badylak SF, Valentin JE, Ravindra AK, et al: Macrophage phenotype as a determinant of biologic scaffold remodeling. Tissue Eng Part A 14: 1835-1842, 2008. 
18. Troidl C, Möllmann H, Nef H, et al: Classically and alternatively activated macrophages contribute to tissue remodelling after myocardial infarction. J Cell Mol Med 13: 3485-3496, 2009.

19. Lanciotti M, Masieri L, Raspollini MR, et al: The role of M1 and M2 macrophages in prostate cancer in relation to extracapsular tumor extension and biochemical recurrence after radical prostatectomy. Biomed Res Int 2014: 486798, 2014

20. Gollapudi K, Galet C, Grogan T, et al: Association between tumor-associated macrophage infiltration, high grade prostate cancer and biochemical recurrence after radical prostatectomy. Am J Cancer Res 3: 523-529, 2013.

21. Comito G, Giannoni E, Segura CP, et al: Cancer-associated fibroblasts and M2-polarized macrophages synergize during prostate carcinoma progression. Oncogene 33: 2423-2431, 2014.

22. Cheng L, Montironi R, Bostwick DG, Lopez-Beltran A and Berney DM: Staging of prostate cancer. Histopathology 60: 87-117, 2012

23. Herroon MK, Rajagurubandara E, Rudy DL, et al: Macrophage cathepsin $\mathrm{K}$ promotes prostate tumor progression in bone. Oncogene 32: 1580-1593, 2013.

24. Murphy BS, Bush HM, Sundareshan V, et al: Characterization of macrophage activation states in patients with cystic fibrosis. J Cyst Fibros 9: 314-322, 2010.

25. Heymann F, Trautwein C and Tacke F: Monocytes and macrophages as cellular targets in liver fibrosis. Inflamm Allergy Drug Targets 8: 307-318, 2009.

26. Desguerre I, Mayer M, Leturcq F, et al: Endomysial fibrosis in Duchenne muscular dystrophy: a marker of poor outcome associated with macrophage alternative activation. J Neuropathol Exp Neurol 68: 762-773, 2009.

27. Mathai SK, Gulati M, Peng X, et al: Circulating monocytes from systemic sclerosis patients with interstitial lung disease show an enhanced profibrotic phenotype. Lab Invest 90: $812-823,2010$
28. Higashi-Kuwata N, Jinnin M, Makino T, et al: Characterization of monocyte/macrophage subsets in the skin and peripheral blood derived from patients with systemic sclerosis. Arthritis Res Ther 12: R128, 2010

29. Huang SP, Bao BY, Wu MT, et al: Impact of prostate-specific antigen (PSA) nadir and time to PSA nadir on disease progression in prostate cancer treated with androgen-deprivation therapy. Prostate 71: 1189-1197, 2011.

30. Chen CH, Hsieh JT, Huang KH, Pu YS and Chang HC: Predictive clinical indicators of biochemical progression in advanced prostate cancer patients receiving Leuplin depot as androgen deprivation therapy. PLoS One 9: e105091, 2014.

31. Petrylak DP, Ankerst DP, Jiang CS, et al: Evaluation of prostate-specific antigen declines for surrogacy in patients treated on SWOG 99-16. J Natl Cancer Inst 98: 516-521, 2006.

32. Armstrong AJ, Garrett-Mayer E, Ou Yang YC, et al: Prostate-specific antigen and pain surrogacy analysis in metastatic hormone-refractory prostate cancer. J Clin Oncol 25: 3965-3970, 2007.

33. Partin AW, Kattan MW, Subong EN, et al: Combination of prostate-specific antigen, clinical stage and Gleason score to predict pathological stage of localized prostate cancer. A multi-institutional update. JAMA 277: 1445-1451, 1997.

34. D'Amico AV, Whittington R, Malkowicz SB, et al: Biochemical outcome after radical prostatectomy, external beam radiation therapy, or interstitial radiation therapy for clinically localized prostate cancer. JAMA 280: 969-974, 1998.

35. Crook J and Ots AF: Prognostic factors for newly diagnosed prostate cancer and their role in treatment selection. Semin Radiat Oncol 23: 165-172, 2013.

36. Phillips JG, Aizer AA, Chen MH, et al: The effect of differing gleason scores at biopsy on the odds of upgrading and the risk of death from prostate cancer. Clin Genitourin Cancer 12: e181-e187, 2014. 